\title{
Analysis of Machine Learning Algorithms for Diagnosis of Diffuse Lung Diseases
}

\author{
Isadora Cardoso ${ }^{1}$ Eliana Almeida ${ }^{1}$ Hector Allende-Cid ${ }^{2}$ Alejandro C. Frery ${ }^{1}$ Rangaraj M. Rangayyan ${ }^{3}$ \\ Paulo M. Azevedo-Marques ${ }^{4}$ Heitor S. Ramos ${ }^{1}$
}

1 Instituto de Computação, Universidade Federal de Alagoas, Maceió, Brazil

2 Escuela de Ingeniería Informatica, Pontificia Universidad Católica de Valparaíso, Valparaíso, Chile

3 Department of Electrical and Computer Engineering, Schulich School of Engineering, University of Calgary, Calgary, Alberta, Canada

${ }^{4}$ Department of Internal Medicine, Ribeirão Preto Medical School,

University of São Paulo, Ribeirão Preto, Brazil

Methods Inf Med 2018;57:e4.

\section{Retraction Notice}

The publisher regrets an error that the above article was published online with an incorrect open access CC BY-NC-ND license.
Address for correspondence Prof. Heitor S. Ramos, Universidade Federal de Alagoas, Institute of Computing, Av. Lourival Melo Mota, S/N, Cidade Universitária, Maceió, Alagoas, 57072900, Brazil (e-mail: heitor@laccan.ufal.br).

For this reason, the article has been retracted and the correct version of the article can be found here: https://doi. org/10.1055/s-0039-1681086. 